\title{
The variation in medial and lateral collateral ligament strain and tibiofemoral forces following changes in the flexion and extension gaps in total knee replacement
}

\author{
A LABORATORY EXPERIMENT USING CADAVER KNEES
}

B. Jeffcote,
R. Nicholls,
A. Schirm,
M. S. Kuster
From Fremantle
Hospital, Fremantle,
Australia

\footnotetext{
B. Jeffcote, BMBS,

FRACS(Orth), Orthopaedic

Surgeon

Department of Orthopaedic Surgery

R. Nicholls, PhD, Research

Fellow

University of Western

Australia, Fremantle

Orthopaedic Unit, Fremantle

Hospital, Alma Street

Fremantle, Western Australia.

A. Schirm, MD, Orthopaedic Surgeon

M. S. Kuster, MD, PhD,

FRACS(Orth), Chairman

Professor

Klinik für Orthopädische

Chirurgie, Kantonsspital 9007

St. Gallen, Switzerland.

Correspondence should be sent to Professor M. S. Kuster; e-mail: Markus.Kuster@kssg.ch
}

(C2007 British Editorial Society of Bone and Joint Surgery doi:10.1302/0301-620X.89B11. $18834 \$ 2.00$

$J$ Bone Joint Surg $[B r]$ 2007;89-B:1528-33.

Received 30 October 2006

Accepted after revision 27 July 2007

\begin{abstract}
Achieving deep flexion after total knee replacement remains a challenge. In this study we compared the soft-tissue tension and tibiofemoral force in a mobile-bearing posterior cruciate ligament-sacrificing total knee replacement, using equal flexion and extension gaps, and with the gaps increased by $2 \mathrm{~mm}$ each. The tests were conducted during passive movement in five cadaver knees, and measurements of strain were made simultaneously in the collateral ligaments. The tibiofemoral force was measured using a customised miniforce plate in the tibial tray. Measurements of collateral ligament strain were not very sensitive to changes in the gap ratio, but tibiofemoral force measurements were. Tibiofemoral force was decreased by a mean of $40 \%$ (SD 10.7) after $90^{\circ}$ of knee flexion when the flexion gap was increased by $2 \mathrm{~mm}$. Increasing the extension gap by $2 \mathrm{~mm}$ affected the force only in full extension. Because increasing the range of flexion after total knee replacement beyond $110^{\circ}$ is a widely-held goal, small increases in the flexion gap warrant further investigation.
\end{abstract}

Flexion often remains limited after total knee replacement (TKR). There have been reports of flexion $\geq 140^{\circ}$ being achieved, ${ }^{1}$ but most studies describe ranges of $105^{\circ}$ to $115^{\circ}$ one year after operation. ${ }^{2-5}$ This range is adequate for most elderly patients in the Western world, but is unlikely to satisfy younger patients, or those for whom kneeling and squatting are important activities. ${ }^{6-8}$ The main factors influencing the post-operative range of flexion identified to date are the pre-operative range, the body mass index, correct sizing of components, patellar tracking, the accuracy of gap balancing and post-operative physiotherapy. ${ }^{9-11}$ This study explores the influence the balance of the flexion/extension gap has on soft-tissue tension in deep flexion.

Insall and $\mathrm{Scott}{ }^{12}$ first recommended balancing knee ligaments by creating equal and rectangular flexion and extension gaps. This recommendation has been generally accepted and several authors have attributed poor clinical results to inadequate balance of these gaps. ${ }^{10,11,13,14}$ The flexion gap may be measured by plain radiography; MR scans are not required. ${ }^{15}$ In practice, the exact balance of the gaps can be difficult to measure, and various intra-operative spreaders or distraction devices have been used to obtain equal gaps. ${ }^{16}$ To date there has been little biomechanical analysis of the influence the balance of the flexion/extension gap has on the forces generated within the tibiofemoral joint or the strains on the periarticular soft tissues. The aim of this study was to quantify the compressive tibiofemoral force and strain patterns in the collateral ligaments in a series of cadaver knees after TKR with equal and unequal flexion and extension gaps.

\section{Materials and Methods}

Five fresh-frozen specimens of the human knee were tested. All had mild to moderate osteoarthritic changes, predominantly in the medial compartment. The femur and tibia were resected $250 \mathrm{~mm}$ from the medial joint line, and all soft tissue except the articular capsule, ligaments, popliteus muscle, and quadriceps tendon was removed.

A simple system was employed to determine the strain in the medial and lateral collateral ligaments (MCL and LCL, respectively). Kirschner $(\mathrm{K})$-wires with a $1.6 \mathrm{~mm}$ diameter were inserted perpendicular to the attachments of the MCL and LCL. The anterior fibres of the MCL were selected for measurement, with the distal K-wire located $3 \mathrm{~mm}$ behind the anterior 


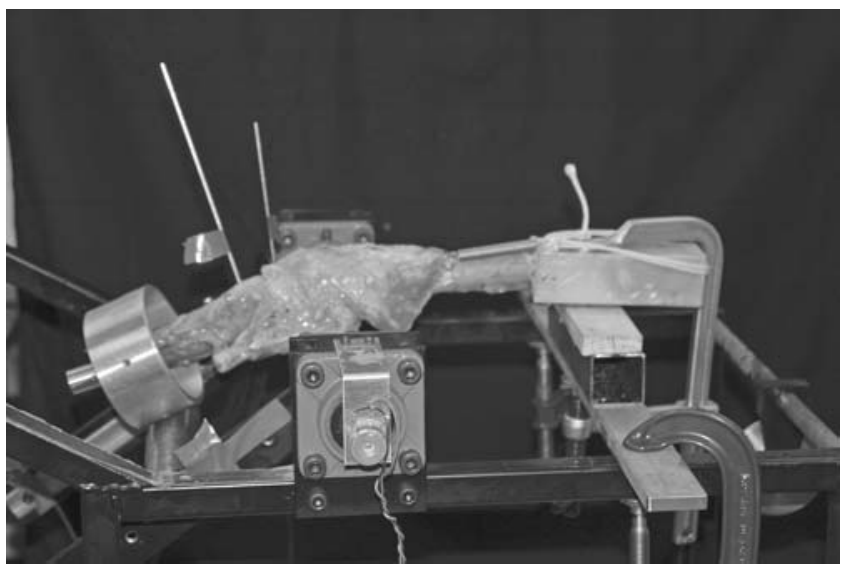

Fig. 1

Photograph of a cadaver knee joint mounted in a customised passive movement rig.

edge of the insertion of the ligament on the tibia. Strain $(\varepsilon)$ was calculated using the engineering strain formula ${ }^{17}(\%)$ :

$$
\varepsilon=\left(\frac{1-1_{0}}{l_{0}}\right)
$$

where $l$ represents the instantaneous length of the ligament (measured with digital calipers accurate to $0.01 \mathrm{~mm}$ ), and $l_{0}$ the reference length, which was the length at full extension for both ligaments prior to implantation of the prosthesis, as previously reported. ${ }^{18}$

In a pilot study, differential variable reluctance transducer (DVRT) strain gauges (Microstrain Inc., Burlington, New England) were used on soft-fixed and fresh-frozen cadaver knees to measure ligament strain, as suggested by Harfe et al. ${ }^{19}$ However, the trials showed inconsistent results beyond $120^{\circ}$ of flexion. We attribute this to the twisting and buckling of the ligaments in deep flexion. Whereas Harfe et $\mathrm{al}^{19}$ presented the strain in the midflexion range, our study interest was beyond $120^{\circ}$ of flexion. Measurements using the $\mathrm{K}$-wire technique were more consistent than the DVRT's throughout the whole range of movement and had the additional advantage of measuring the behaviour of the ligament as a whole, rather than individual fibre bundles.

Each knee was mounted in a customised passive movement rig (Fig. 1) designed to apply a passive flexionextension moment to the tibia with the femur fixed. The rig also permitted the normal rotation of the tibia during movement. It aimed to mimic the surgical environment where the knee is passively flexed by the surgeon with the patient anaesthetised. A spring sutured to the quadriceps tendon was calibrated such that it exerted no force at full extension but gradually came into play as the knee flexed, reaching a maximum force of $40 \mathrm{~N}$ at $150^{\circ}$ of flexion. The purpose of the spring was to reproduce the passive stretch-

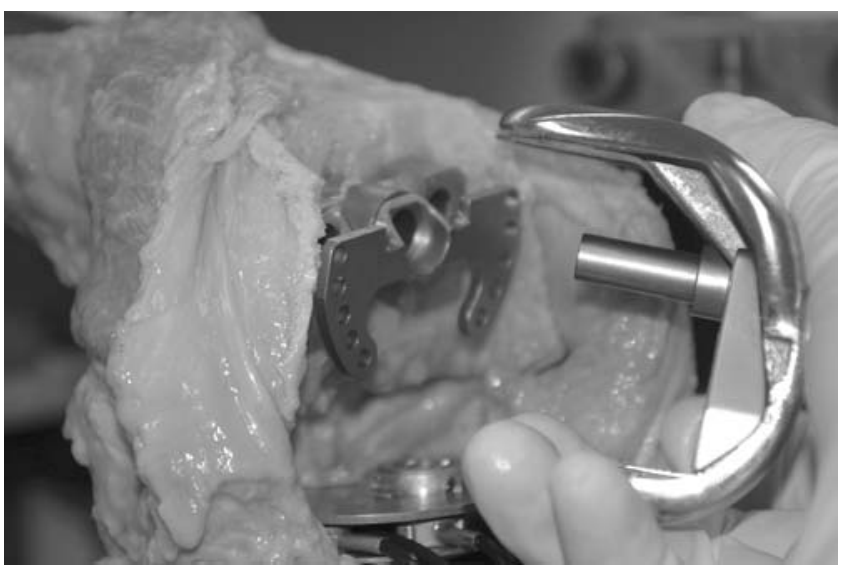

Fig. 2

Photograph of the adjustable low contact stress femoral component in situ.

ing of the extensor mechanism which generates force across the tibiofemoral articulation in flexion. As there is minimal hamstring tension during knee flexion in the anaesthetised patient, no hamstring force was simulated.

Each joint was pre-conditioned in the rig by the application of ten cycles of flexion from full extension to $150^{\circ}$ (at $30^{\circ}$ per second). The angle of flexion of the joint was determined using a calibrated rotary-angle potentiometer attached to the rig. In each specimen, measurements of the length of the MCL and LCL were obtained at $15^{\circ}$ increments between $0^{\circ}$ and $150^{\circ}$ flexion. These measurements were then repeated with two further cycles of flexion.

The knee was then removed from the rig and a medial parapatellar arthrotomy with osteotomy of the tibial tuberosity was performed to gain access to the knee joint. The osteotomy was fixed with two small fragment screws, and the arthrotomy closed with 1-vicryl sutures. The Low Contact Stress rotating platform instrumentation (LCS, DePuy, Warsaw, Indiana) was used to prepare the bone cuts. Both cruciate ligaments were resected. The femur was divided using an intramedullary guide in $5^{\circ}$ of valgus. Tibial resection was performed with a $7^{\circ}$ posterior slope referenced from an intramedullary guide, as the ankle was not present. There was no significant coronal bowing of any of the tibiae. The width of the flexion and extension gaps was measured using digital callipers while applying a $100 \mathrm{~N}$ distraction force to the distal tibia in full extension and $90^{\circ}$ of flexion.

In this study two femoral components were used. The first was the standard LCS femoral trial, which was press-fitted to the divided distal femur. This component was used to obtain measurements of baseline force and ligament strain for a standard implantation technique with balanced flexion and extension gaps (balanced gap (BG) series). The second component was a custom-designed LCS femoral component mounted on an intramedullary rod (Fig. 2). The geometry of the articular surface was identical to that of the trial 


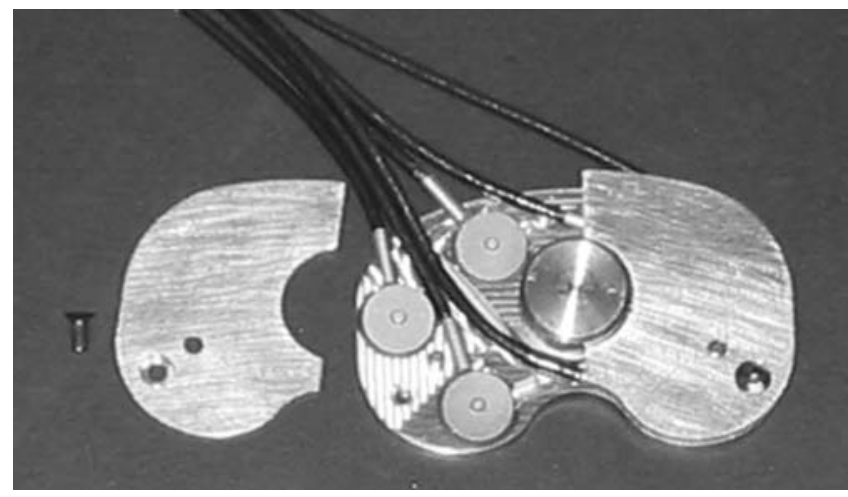

Fig. 3

Photograph of a customised miniature force platform designed for measurement of uniaxial compressive tibiofemoral force in vitro.

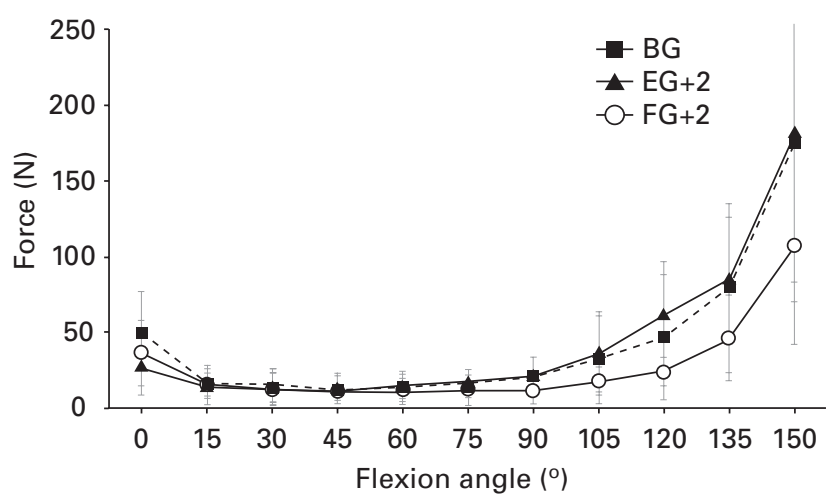

Fig. 4

Graph showing the mean values for tibiofemoral force during flexion for the balanced gap (BG), extension gap $+2 \mathrm{~mm}(E G+2)$ and flexion gap + $2 \mathrm{~mm}(\mathrm{FG}+2)$. The error bars show the range of results at each flexion angle across five specimens.

ligament strain obtained at $15^{\circ}$ increments between $0^{\circ}$ and $150^{\circ}$ knee flexion. allowing accurate adjustment of the extension gap. The adjustable femoral component was implanted aligning the component with the previous femoral cuts. In order to increase the extension gap (EG) by $2 \mathrm{~mm}$ (EG+2 series) the adjustable femoral component was translated $2 \mathrm{~mm}$ proximally. In order to increase the flexion gap (FG) by $2 \mathrm{~mm}$ (FG+2 series) the adjustable femoral component was translated $2 \mathrm{~mm}$ distally while the tibial component was also simultaneously translated $2 \mathrm{~mm}$ distally, with the effect of increasing the flexion gap by $2 \mathrm{~mm}$ relative to the extension gap.

In place of the standard LCS tibial tray, a miniature force platform was implanted (Fig. 3). This was designed, calibrated and validated in our laboratory, and was constructed to allow the trial LCS rotating platform insert to rest on the platform and rotate a central hub. A brass baseplate was constructed to house six load sensors arranged in triangular arrays in the medial and lateral compartments. These sensors allow the measurement of compressive loads (tibiofemoral force) in each compartment individually. The system was linked to a PC notebook computer via a National Instruments SC-2345 connector block and 6024E 12-bit data acquisition card (National Instruments, Austin, Texas). The force platform was implanted by mounting it on an intramedullary rod which could be adjusted to translate the platform proximally or distally. The rod was fixed within the tibial shaft using three screws and a wire stirrup. The force platform was stabilised on the cut tibial surface using metal shims, small metal wedges $10 \mathrm{~mm}$ in length and $2 \mathrm{~mm}$ maximum height, manufactured at the Fremantle Hospital Biomedical Services Department.

After implanting the components, an identical loading regimen was undertaken for each condition $(\mathrm{BG}, \mathrm{EG}+2$, $\mathrm{FG}+2$ ). After pre-conditioning, two cycles of flexion were performed with measurements of tibiofemoral force and

\section{Results}

Tibiofemoral force. The mean tibiofemoral force measurements obtained from the force platform for the three measurement series are shown in Figure 4. The main variations of force occurred in full extension and beyond $90^{\circ}$ of knee flexion.

The mean tibiofemoral force in the balanced gap group was $50 \mathrm{~N}$ (SD 26.6 for five specimens) at full extension. This was a starting point chosen to reproduce the force that might be expected in a TKR during implantation. The mean force between $15^{\circ}$ and $75^{\circ}$ of flexion in the BG series was 15.5 N (SD 9.6), before rising in an exponential manner to a peak at a mean of $175 \mathrm{~N}$ (SD 104) at $150^{\circ}$ of flexion.

For the FG+2 group the mean force at full extension was slightly less at $37 \mathrm{~N}$ (SD 18.9) and remained less than $15 \mathrm{~N}$ (SD 9.7) until $90^{\circ}$ of flexion. Thereafter, the force again rose exponentially but less steeply than in the BG series, peaking at a mean of $107 \mathrm{~N}$ (SD 65.1) at full flexion. This amounted to a mean force reduction of $40 \%$ beyond $90^{\circ}$ of knee flexion.

The mean force at full extension was also lower for the $\mathrm{EG}+2$ group at $27 \mathrm{~N}$ (SD 18.6), but the force from $15^{\circ}$ of flexion to $150^{\circ}$ of flexion was similar to the BG series.

Collateral ligament strain. The measurements of collateral ligament length prior to implantation of a prosthesis were used as a baseline. The mean strains in the anterior MCL and LCL are shown in Figures 5 and 6.

The anterior portion of the MCL recorded very little change in length throughout the flexion range in the intact knee. The mean peak strain was $1.7 \%$ (SD 2.4) at $90^{\circ}$ flexion, with a mean minimum strain of $-0.8 \%$ (SD 3.9) at $150^{\circ}$. The LCL showed a very different strain pattern, with little change in length over the first $60^{\circ}$ of flexion followed by progressive loosening to a mean minimum strain of $-15.3 \%$ (SD 4.5 ) at $150^{\circ}$. 


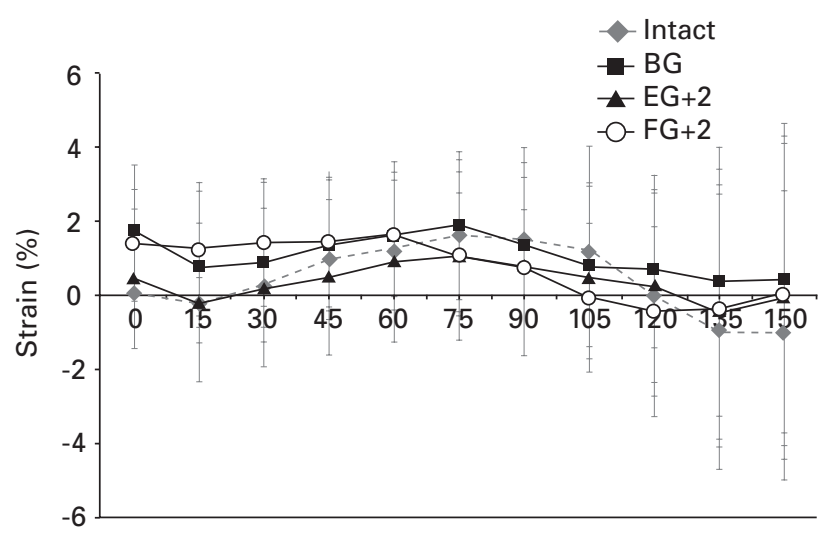

Flexion angle $\left({ }^{\circ}\right)$

Fig. 5

Graph showing the mean values for medial collateral ligament strain during passive flexion for three total knee replacements compared with the baseline (intact knee strain) (BG, balanced gap; $E G+2$, extension gap +2 $\mathrm{mm} ; \mathrm{FG}+2$, flexion gap $+2 \mathrm{~mm})$. The error bars show the range of results at each flexion angle across five specimens.

After implantation of the components with BG, the mean anterior MCL strain at full extension and full flexion was slightly increased compared with the measurements in the intact knee $(1.6 \%$ strain, SD 1.8$)$. The LCL was slightly looser than the pre-implantation measurements at full extension with a mean of $-1.5 \%$ (SD 2.1) strain, but as the knee flexed the slackening of the LCL was delayed to $90^{\circ}$ of knee flexion and less marked than in the intact knee. The mean minimum strain was $-7.0 \%$ (SD 3.8) at full flexion after implantation of the components.

In the FG+2 group, the ligament strains were very similar to those in the BG group. In the $\mathrm{EG}+2$ group the ligament strains were approximately $2 \%$ lower near extension than in the balanced knee. This was the comparison of the mean strain at extension in the $\mathrm{BG}$ group compared with the mean strain in the EG+2 group. Otherwise no obvious differences were detected.

\section{Discussion}

We believe this is the first study to examine the tibiofemoral forces and collateral ligament strain for variations in flexion and extension gaps. The behaviour of the collateral ligament strain was not greatly different with the variations, but the changes in tibiofemoral force were. The non-linear stress-strain behaviour and complex anatomy of the knee ligaments, combined with the limitations of the strain measuring device, made collateral ligament strain measurement less sensitive than with force transducers to changes in soft-tissue tension. The force transducers were able to detect force generated not only from the collaterals but also from tension in the extensor mechanism, retinaculum and capsule, allowing a more comprehensive measurement of the soft-tissue balance. The effect

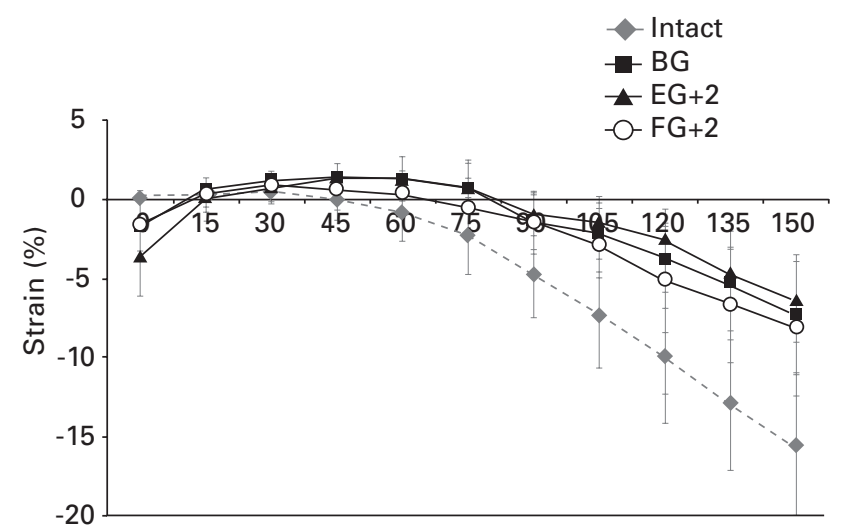

Flexion angle $\left({ }^{\circ}\right)$

Fig. 6

Graph showing the mean values for lateral collateral ligament strain during passive flexion for the total knee replacements compared with the baseline (intact knee strain) (BG, balanced gap; $E G+2$, extension gap + $2 \mathrm{~mm} ; \mathrm{FG}+2$, flexion gap $+2 \mathrm{~mm}$ ). The error bars show the range of results at each flexion angle across five specimens.

of the mobile-bearing articulation on the collateral ligament strain in this study is difficult to identify clearly, but we believe that the relative insensitivity of the measurements of ligament strain compared with the force transducers would apply equally to fixed- as well as mobilebearing prostheses.

Precise balancing of flexion and extension gaps is at times difficult to achieve at operation. ${ }^{20}$ Surgeons may have to accept slight discrepancies in balance and it is, therefore, important to know the effects of small variations in gap ratio on the soft-tissue tension.

The results of the balanced gap group show that the comprehensive force within the cadaver knees decreased in early flexion, remained low throughout mid-flexion, and then increased exponentially after $90^{\circ}$. When the extension gap was increased by $2 \mathrm{~mm}$ relative to the flexion gap, there was a decrease in the tibiofemoral force in full extension only. Beyond $15^{\circ}$ of flexion the force and ligament strains were essentially the same as in the balanced gap group. When the flexion gap was increased by $2 \mathrm{~mm}$ relative to the extension gap the results were markedly different. The tibiofemoral force in full extension was hardly influenced (mean $37 \mathrm{~N}$ vs $50 \mathrm{~N}$ ), and during mid-flexion the tibiofemoral force was similar to the other two groups. Beyond $90^{\circ}$ of flexion, however, the force was reduced by a mean of $40 \%$. This finding suggests that small variations in the flexion or extension gaps have little effect on the soft-tissue tension between $15^{\circ}$ and $100^{\circ}$ of flexion, which is the range of movement achieved by most patients after TKR. However in deeper flexion, ligament tension is sensitive to changes in the flexion gap. Increasing the flexion gap by as little as $2 \mathrm{~mm}$ may have benefits in terms of reduced generation of tibiofemoral force beyond $90^{\circ}$ of flexion. 
Although the benefits of a balanced flexion and extension gap are based on sound theory, there are no published biomechanical data to confirm this recommendation. There are good reasons to question this traditional approach. Several studies, including this one, have shown a non-isometric behaviour of the LCL, ${ }^{21}$ and to a lesser extent the MCL. ${ }^{19,22}$ Hence, balancing the gaps at $0^{\circ}$ and $90^{\circ}$ of flexion will not necessarily provide ideal soft-tissue tension in deeper flexion. Indeed, inferring from the present data, an increased flexion gap of $2 \mathrm{~mm}$ reduces soft-tissue tension by approximately $40 \%$ in deep flexion. Although equal flexion and extension gaps will obtain knee flexion up to approximately $110^{\circ}$, it seems advantageous to increase the flexion gap slightly when deep knee flexion is a goal. There is also clinical evidence in the literature to support this hypothesis. In a clinical trial with bilateral TKRs it was shown that patients consistently preferred the laxer knee, which tended to provide an increased range of movement. ${ }^{23}$ Several other authors have suggested that an increased flexion gap might improve the post-operative range of movement. ${ }^{24-26}$

Our study showed that a looser flexion gap does decrease soft-tissue tension beyond $120^{\circ}$ of knee flexion. This is in contrast to the work of Bellemans et al, ${ }^{27}$ who emphasised the importance of a sufficient posterior condylar offset. Whereas reduced condylar offset can induce posterior tibiofemoral impingement, an increased offset tightens the flexion gap and increases soft-tissue tension in flexion. As both mechanisms seem to restrict movement, a balance between condylar offset and soft-tissue tension in flexion must be achieved. Furthermore, femoral rollback during flexion can reduce the influence of the posterior condylar offset on tibiofemoral impingement. Indeed, in the study by Bellemans et $\mathrm{al}^{27}$ the kinematic analysis demonstrated a parodoxical roll forward of the femur in flexion. Hence, the patients in their series depended on a high condylar offset to avoid impingement, whereas patients with sufficient femoral rollback are less reliant. In most current designs of TKR the tibial insert is concave. In order to allow the femur to roll back the ligaments must loosen in flexion to accommodate the necessary superior translation. This can be obtained by an increased tibial slope and a slightly looser flexion gap. Kinematic in vitro and in vivo analyses are necessary to give further insight into the complex and at times conflicting interactions between the amount of condylar offset, tibial slope, soft-tissue tension, and their influence on femoral rollback and tibiofemoral impingement. The lack of kinematic data is therefore a limitation of the current study.

The potential disadvantage of increasing the flexion gap is that of instability in flexion, which in some cases can require revision surgery. ${ }^{14}$ However, we do not believe that symptomatic instability in flexion is likely when increasing the gap by $2 \mathrm{~mm}$, but to consistently obtain a $2 \mathrm{~mm}$ increased flexion gap and to avoid instability, optimal tension is needed.
We chose to use a mobile-bearing posterior cruciate ligament (PCL) sacrificing prosthesis in this study. Although this is not representative of all TKR designs, it is a commonly-used implant with a long and successful track record. ${ }^{28}$ The effects of a relative increase in the flexion gap are unlikely to be specific to this individual design of prosthesis. We anticipate that TKR designs with a concave tibial insert, such as most PCL-sacrificing, PCL-substituting and medial pivot designs, may demonstrate an increased range of flexion with a looser flexion gap. The effect of an increased flexion gap on PCL-retaining designs, which generally have flatter tibial inserts, is difficult to anticipate from this study. A recent study showed results that support a relatively loose flexion gap also for PCL-retaining knees. $^{29}$

This study was only able to evaluate compressive tibiofemoral force generation in the unloaded cadaver knees. It does not take account of the generation of shear forces within the knee, which could be significant, especially in a lax or unstable implantation. Also, the compressive forces are low compared with the forces expected within the knee during normal walking. However, the generation of tibiofemoral force in deep flexion is a reflection of the soft-tissue tension around the knee. This is likely to be a restriction to flexion in the knee replacement in vivo. Given that increasing the flexion range of TKRs is a widely-held goal, small increases in the flexion gap relative to the extension gap may be a method worth investigating further.

No benefits in any form have been received or will be received from a commercial party related directly or indirectly to the subject of this article.

\section{References}

1. Kim YH, Sohn KS, Kim JS. Range of motion of standard and high-flexion posterior stabilized total knee prostheses: a prospective, randomized study. J Bone Joint Surg [Am] 2005;87-A:1470-5

2. Huang CH, Ma HM, Lee YM, Ho FY. Long-term results of low contact stress mobilebearing total knee replacements. Clin Orthop 2003;416:265-70.

3. Schurman DJ, Rojer DE. Total knee arthroplasty: range of motion across five systems. Clin Orthop 2005;430:132-7.

4. Epinette JA, Manley MT. Hydroxyapatite-coated total knee replacement: clinical experience at 10 to 15 years. J Bone Joint Surg [Br] 2007;89-B:34-8.

5. Cross MJ, Parish EN. A hydroxyapatite-coated total knee replacement: prospective analysis of 1000 patients. J Bone Joint Surg [Br] 2003;87-B:1073-6.

6. Noble PC, Gordon MJ, Weiss JM, et al. Does total knee replacement restore normal knee function? Clin Orthop 2005;431:157-65.

7. Palmer SH, Servant CT, Maguire J, Parish EN, Cross MJ. Ability to kneel after total knee replacement. J Bone Joint Surg [Br] 2002;84-B:220-2.

8. Park KK, Chang CB, Yang KG, Seong SC. Correlation of maximum flexion with clinical outcome after total knee replacement in Asian patients. J Bone Joint Surg [Br] 2007;89-B:604-8.

9. Ritter MA, Harty LD, Davis KE, Meding JB, Berend ME. Predicting range of motion after total knee arthroplasty: clustering, log-linear regression, and regression tree analysis. J Bone Joint Surg [Am] 2003;85-A:1278-85.

10. Kim J, Nelson CL, Lotke PA. Stiffness after total knee arthroplasty: prevalence of the complication and outcomes of revision. J Bone Joint Surg [Am] 2004;86-A:147984.

11. Gandhi R, de Beer J, Leone J, et al. Predictive risk factors for stiff knees in total knee arthroplasty. J Arthroplasty 2006;21:46-52.

12. Insall JN, Scott WN. Surgery of the knee. Third ed. New York: Churchill Livingstone, 2001.

13. McAuley JP, Engh GA. Constraint in total knee arthroplasty: when and what? J Arthroplasty 2003;18(Suppl 1):51-4. 
14. Pagnano MW, Hanssen AD, Lewallen DG, Stuart MJ. Flexion instability after primary posterior cruciate retaining total knee arthroplasty. Clin Orthop 1998;356:39-

15. Tokuhara Y, Kadoya Y, Kanekasu K, et al. Evaluation of the flexion gap by axial radiography of the distal femur. J Bone Joint Surg [Br] 2006;88-B:1327-30.

16. Mihalko WM, Whiteside LA, Krackow KA. Comparison of ligament balancing technique during total knee arthroplasty. J Bone Joint Surg [Am] 2003;85-A(Suppl 4):132-5.

17. Cerulli G, Benoit DL, Lamontagne M, Caraffa A, Liti A. In vivo anterior cruciate ligament strain behaviour during a rapid deceleration movement: case report. Knee Surg Sports Traumatol Arthrosc 2003;111:307-11.

18. Arms S, Boyle J, Johnson R, Pope M. Strain measurement in the medial collateral ligament of the human knee: an autopsy study. J Biomechanics 1983;7:491-6.

19. Harfe DT, Chuinard CR, Espinoza LM, Thomas KA, Solomonov M. Elongation patterns of the collateral ligaments of the human knee. Clin Biomech (Bristol, Avon) 1998;13:163-75

20. Griffin FM, Insall JN, Scuderi GR. Accuracy of soft tissue balancing in total knee arthroplasty. J Arthroplasty 2000;15:970-3.

21. Meister BR, Michael SP, Moyer RA, Kelly JD, Schneck CD. Anatomy and kinematics of the lateral collateral ligament of the knee. Am J Sports Med 2000;28:86978.
22. Gardiner JC, Weiss JA. Experimental testing and computational modelling to determine the stress-strain distribution in the human medial collateral ligament. Trans Orth Res Soc 1998:23:1027.

23. Kuster MS, Bitschnau B, Votruba T. Influence of collateral ligament laxity on patient satisfaction after total knee arthroplasty: a comparative bilateral study. Arch Orthop Trauma Surg 2004;124:415-17.

24. Edwards E, Miller J, Chan KH. The effect of postoperative collateral ligament laxity in total knee arthroplasty. Clin Orthop 1988;236:44-51.

25. Chiu KY, Ng TP, Tang WM, Yau WP. Review article: knee flexion after total knee arthroplasty. J Orthop Surg (Hong Kong) 2002;10:194-202.

26. Asano H, Hoshino A, Wilton TJ. Soft tissue tension in total knee arthroplasty. J Arthroplasty 2004;19:558-61.

27. Bellemans J, Banks S, Victor J, Vandenneucker H, Moemans A. Fluoroscopic analysis of the kinematics of deep flexion in total knee arthroplasty: influence of posterior condylar offset. J Bone Joint Surg [Br]2002;84-B:50-3.

28. Sorrells RB, Capps SG. Clinical results of primary low contact stress cementless total knee arthroplasty. Orthopaedics 2006;29 (9 Suppl):542-4.

29. Schuster AJ, von Roll A, Wyss T. Midterm results and stability measurements after total knee arthroplasty using the ligament balancing technique: a prospective study. Presented at EFORT Congress 2002. http://www.efort.org/e/cd2007/f166.pdf (date last accessed 9 October 2007). 